\title{
BMJ Open Admission glucose level and short-term mortality in older patients with acute myocardial infarction: results from the KORA Myocardial Infarction Registry
}

\author{
Temur Mamadjanov (D) ,1,2 Konstantinos Volaklis, ${ }^{2}$ Margit Heier, ${ }^{3,4}$ Dennis Freuer, ${ }^{2}$ \\ Ute Amann, ${ }^{5}$ Annette Peters, ${ }^{3}$ B Kuch, ${ }^{6}$ Christian Thilo, ${ }^{7}$ Jakob Linseisen, ${ }^{2,5}$ \\ Christa Meisinger ${ }^{2}$
}

To cite: Mamadjanov T, Volaklis K, Heier M, et al. Admission glucose level and short-term mortality in older patients with acute myocardial infarction: results from the KORA Myocardial Infarction Registry. BMJ Open 2021;11:e046641. doi:10.1136/ bmjopen-2020-046641

- Prepublication history for this paper is available online. To view these files, please visit the journal online (http://dx.doi. org/10.1136/bmjopen-2020046641).

Received 11 November 2020 Accepted 20 May 2021

Check for updates

(C) Author(s) (or their employer(s)) 2021. Re-use permitted under CC BY-NC. No commercial re-use. See rights and permissions. Published by BMJ.

For numbered affiliations see end of article.

Correspondence to Temur Mamadjanov; temurmamadjanov@gmail.com

\section{ABSTRACT}

Study objectives To investigate the association between admission blood glucose levels and 28-day mortality as well as in-hospital complications in older patients with incident acute myocardial infarction (AMI) undergoing modern treatment.

Methods From a German population-based regional MI registry, 5530 patients (2016 women), aged 65-84 years, hospitalised with an incident AMI between 1 January 2009 and 31 December 2016 were included in the study. Multivariable logistic regression models were used to assess the associations between admission blood glucose and 28-day mortality as well as in-hospital complications after AMI. Analyses stratified according to age, diabetes and type of infarction (ST-elevation MI (STEMI)/non-STEMI) were conducted.

Results The adjusted ORs for the association between admission blood glucose and 28-day mortality in youngold (65-74 years) and old (75-84 years) patients with AMl were $1.40(95 \% \mathrm{Cl}: 1.21$ to 1.62$)$ and $1.21(95 \%$ Cl: 0.98 to 1.50 ) per 1 SD increase in admission blood glucose, respectively. Furthermore, higher admission blood glucose was related to case fatality irrespective of the diabetes status and type of infarction only in the under- 75 group. For the patients aged $75-84$ years, it was only true for those without diabetes and STEMI. Admission blood glucose was also associated with major cardiac complications in both age groups.

Conclusion Admission blood glucose was significantly associated with 28-day case fatality in patients with AMI aged $65-74$ years but not $75-84$ years; furthermore, in both age groups there was an increased risk of major complications. It seems that admission glucose may play a rather minor role in terms of case fatality in higher aged patients with AMI.

\section{INTRODUCTION}

Elevated admission blood glucose levels are common in patients hospitalised for acute myocardial infarction (AMI); the prevalence of admission hyperglycaemia in
Strengths and limitations of this study

- This study was observational and was limited to German patients aged 65-84 years old with incident acute myocardial infarction.

- The analysis was limited to admission blood glucose values only and it cannot be ruled out that some patients with hyperglycaemia without a history of diabetes are true diabetes cases who have not been diagnosed before

- Multivariable analysis was adjusted for several risk factors, thus residual confounding cannot be entirely excluded.

- Data were collected within the framework of a population-based myocardial infarction registry.

- Important risk factors such as comorbidities, inhospital treatment and complications were included in the analysis.

epidemiological studies for these patients ranges from $40 \%$ to $>58 \% .{ }^{12}$ Several studies and meta-analyses further suggested that hyperglycaemia on admission is an independent risk factor for adverse outcomes and mortality among patients hospitalised with AMI. $^{34}$

Timmer et a $\tilde{p}^{-8}$ demonstrated that a higher glucose level on admission is independently associated with increased sensitivity to ischaemia reperfusion injury such as impaired initial flow in the infarct-related artery. Blood glucose level was also described as an independent prognostic factor for impaired microvascular function or the no-reflow phenomenon. $^{9}$ In addition, some studies showed that patients with hyperglycaemia have a higher Killip class and thus mortality risk. ${ }^{10}$ Moreover, a larger infarct size and worse left ventricular (LV) function were linked to a higher glucose level, ${ }^{11}$ and an addition of blood glucose levels improves the predictive 
ability of the Global Registry of Acute Coronary Events risk score. ${ }^{12} 13$

The majority of the existing studies were conducted in the pre-reperfusion era, ${ }^{3}{ }^{14-16}$ were focused on patients with or without diabetes ${ }^{17} 18$ or included the whole spectrum of acute coronary syndromes in their analysis. ${ }^{19}$ So far, only a few studies examined the association between admission blood glucose levels and short-term outcomes (including in-hospital mortality and cardiac complications) in older people. ${ }^{20}$ Furthermore, the association between admission glucose in certain subgroups of older patients with AMI is missing so far. Therefore, the aim of this study including all non-selected hospitalised cases with incident AMI was to investigate the association of admission glucose on 28-day case fatality and cardiac complications in patients aged 65-84 years old undergoing non-invasive and invasive therapy. Analyses stratified according to diabetes, age and type of infarction were conducted to determine the importance of admission blood glucose for the short-term prognosis of certain patient subgroups with AMI.

\section{METHODS}

\section{Study design and data source}

Data for the present observational study came from the population-based KORA (Cooperative Health Research in the Region of Augsburg) Myocardial Infarction Registry (Bavaria, Germany), which was implemented in October 1984 as part of the WHO MONICA (Monitoring Trends and Determinants in Cardiovascular Disease) Project. Since then all cases of fatal and non-fatal AMI occurring among the residents of the study area (city of Augsburg and two adjacent counties) aged 25-74 years old, who were admitted to one out of eight hospitals in the study area have been continuously registered. The registry was included into the KORA framework when the MONICA Project was terminated in 1995. Detailed information on methods of case identification, diagnostic classification of events and quality control of the data has been described in previous publications. ${ }^{21-23}$ Diagnostic criteria for AMI case identification were adapted to the joint statement of the European Society of Cardiology and American College of Cardiology and applied since 2001. ${ }^{24}$ From 2009 onwards, the registry was extended for the elderly up to 84 years.

\section{Data collection and measurements}

Patients with AMI, who have survived for at least 24 hours after hospitalisation, were interviewed by specially trained nurses using a standardised questionnaire. Information on sociodemographic data, acute symptoms, cardiovascular risk factors and history of several diseases was collected. Diabetes status (yes/no) was based on what was known on admission only. Data on AMI characteristics, drug treatment before and during hospital stay, medication use at discharge, in-hospital adverse events, including ventricular fibrillation, cardiogenic shock, cardiac arrest, recurrent MI, and pulmonary oedema, were provided by chart review. Additionally, laboratory parameters including the first blood glucose level at admission (referred as admission glucose level), ECG data and the process of care in hospital were also determined. The kind of reperfusion therapy (thrombolysis, percutaneous coronary intervention (PCI) and coronary artery bypass grafting $(\mathrm{CABG}))$ was documented.

\section{Study population}

Between 1 January 2009 and 31 December 2016, a total of 7681 patients aged 65-84 years were admitted to one of the hospitals in the study region due to an AMI. Of those, 1803 patients had a reinfarction and 9 patients had missing information on infarction history and were therefore excluded. Furthermore, we excluded 255 patients without data on admission glucose level and 84 patients with missing covariates information. This resulted in a total of 5530 patients ( 3514 men, 2016 women) with incident AMI for analysis.

\section{Patient and public involvement}

Patients and the public were not involved in the research process.

\section{Outcomes}

The primary endpoint of the study was case fatality within 28 days. A multiple logistic regression model was used to assess the association between the first admission glucose level and 28-day case fatality (yes/no). The secondary endpoint was a combined endpoint of in-hospital complications including cardiac arrest, recurrent infarction, pulmonary oedema, cardiogenic shock, ventricular tachycardia, ventricular bradycardia and ventricular fibrillation.

\section{Statistical analysis}

Continuous data were expressed as mean values and SD as well as median and IQR (25th and 75th quintile) in case of non-normal distribution. Categorical data were described with absolute values and percentages. $\mathrm{X}^{2}$ test was used to test differences in prevalence. The two-sided Welch's t-test was used to compare means.

Due to the large number of missing values presented in table 1, we used multiple imputation before regression. Since the missing mechanism was not completely at random, this approach minimised bias of the effect estimates and increased statistical power. Multivariable analyses were performed for the whole sample and also stratified by age groups (65-74/75-84 years), diabetes status (yes/no) and type of infarction (ST-elevation MI (STEMI) / non-STEMI (NSTEMI)) using forward stepwise logistic regression to identify variables independently associated with 28-day case fatality (yes/no) after AMI. The variables age (only in the analysis including the total sample) and sex were forced into each model during the variable selection procedure. The significance criterion for staying in the final model was chosen as $p<0.05$. The association between admission blood glucose level and 
Table 1 Characteristics of the patients with AMI by age groups

\begin{tabular}{|c|c|c|c|c|}
\hline & \multicolumn{2}{|l|}{ Age groups } & \multirow[b]{2}{*}{$P$ value } & \multirow{2}{*}{$\begin{array}{l}\text { Total sample } \\
(\mathrm{n}=5530)\end{array}$} \\
\hline & $65-74(n=3709)$ & $75-84(n=1821)$ & & \\
\hline Female sex & $1185(31.9 \%)$ & $831(45.6 \%)$ & $<0.001$ & $2016(36.5 \%)$ \\
\hline Lipid disorder & $2238(60.3 \%)$ & $906(49.8 \%)$ & $<0.001$ & $3144(56.9 \%)$ \\
\hline \multicolumn{5}{|l|}{ Smoking status } \\
\hline Ex-smoker & $1245(33.6 \%)$ & $533(29.3 \%)$ & & $1778(32.2 \%)$ \\
\hline Never smoker & $1291(34.8 \%)$ & $792(43.5 \%)$ & & $2083(37.7 \%)$ \\
\hline Missing & $499(13.5 \%)$ & $375(20.6 \%)$ & & $874(15.8 \%)$ \\
\hline Glucose level on admission (mg/dL) (median (IQR)) & $92.0(71.0)$ & $97.0(71.0)$ & 0.521 & $94.0(70.0)$ \\
\hline Peak glucose level (mg/dL) (median (IQR)) & $98.0(60.0)$ & $95.0(81)$ & $<0.001$ & $97.0(87.0)$ \\
\hline Missing & 987 (26.6\%) & $288(15.8 \%)$ & & $1275(23.1 \%)$ \\
\hline Diabetes & $1376(37.1 \%)$ & 721 (39.6\%) & 0.077 & 2097 (37.9\%) \\
\hline \multicolumn{5}{|l|}{ Type of Infarction } \\
\hline STEMI & $1189(32.1 \%)$ & $440(24.2 \%)$ & $<0.001$ & 1629 (29.5\%) \\
\hline NSTEMI & 1967 (53.0\%) & 898 (49.3\%) & & $2865(51.8 \%)$ \\
\hline Bundle branch block & $316(8.5 \%)$ & 220 (12.1\%) & & $536(9.7 \%)$ \\
\hline Not defined & 237 (6.4\%) & 263 (14.4\%) & & $500(9.0 \%)$ \\
\hline Typical symptoms & $2896(78.1 \%)$ & $1218(66.9 \%)$ & & $4114(74.4 \%)$ \\
\hline
\end{tabular}

AMI, acute myocardial infarction; LVEF, left ventricular ejection fraction; NSTEMI, non-ST-elevation myocardial infarction; STEMI, ST-elevation myocardial infarction.

the primary endpoint was adjusted for sex and age in the first model. The second model included previous factors and any reperfusion therapy (yes/no), treatment with ACE inhibitor (yes/no), beta-blocker (yes/no), lipidlowering drug (yes/no), antiplatelets during hospital stay (yes/no), insulin (yes/no), cardiac arrest during hospitalisation (yes/no), any other complication during hospital stay (recurrent infarction, ventricular fibrillation, ventricular tachycardia, bradycardia, pulmonary oedema and cardiogenic shock), and diabetes (yes/no).

In logistic regression analysis investigating the association between admission blood glucose level and the secondary endpoint, the first model included admission blood glucose, age and sex. The second model was adjusted additionally for diabetes (yes/no), any reperfusion therapy (yes/no), treatment with ACE inhibitor (yes/ no), beta-blocker (yes/no), lipid-lowering drug (yes/no), antiplatelets during hospital stay (yes/no) and insulin (yes/no). ORs and 95\% CI interval were computed per 1 SD increase of admission blood glucose level.

We conducted a formal test to identify an interaction with sex, age, diabetes and MI type. The test showed significant interaction with age and diabetes. Due to a significant interaction with age, the sample was stratified into two age groups: 'young-old' patients (65-74 years) and 'old' patients (75-84 years) (figure 1). In addition, stratified analyses were conducted for patients with and without diabetes, and for patients with STEMI/NSTEMI.

We used restricted cubic splines with different numbers of knots for testing the linearity assumption of the appropriate multivariable logistic model. For all investigations, a significance level of 5\% was applied. Analyses were performed using R V.3.5.2.

\section{RESULTS}

In total, the study sample consisted of 5530 women and men aged 65-84 years. There were $292(7.9 \%)$ deaths within 28 days among 3709 patients aged 65-74 years and $209(11.5 \%)$ deaths among 1821 patients aged 75-84 years. The median admission glucose level was $94.0 \mathrm{mg}$ / $\mathrm{dL}$ (IQR $68.0-138.0 \mathrm{mg} / \mathrm{dL}$ ) and $37.9 \%$ of the patients in the total sample had known diabetes.

The baseline characteristics of the patients according to the age groups are shown in table 1 . The older age group was associated with a higher proportion of female 


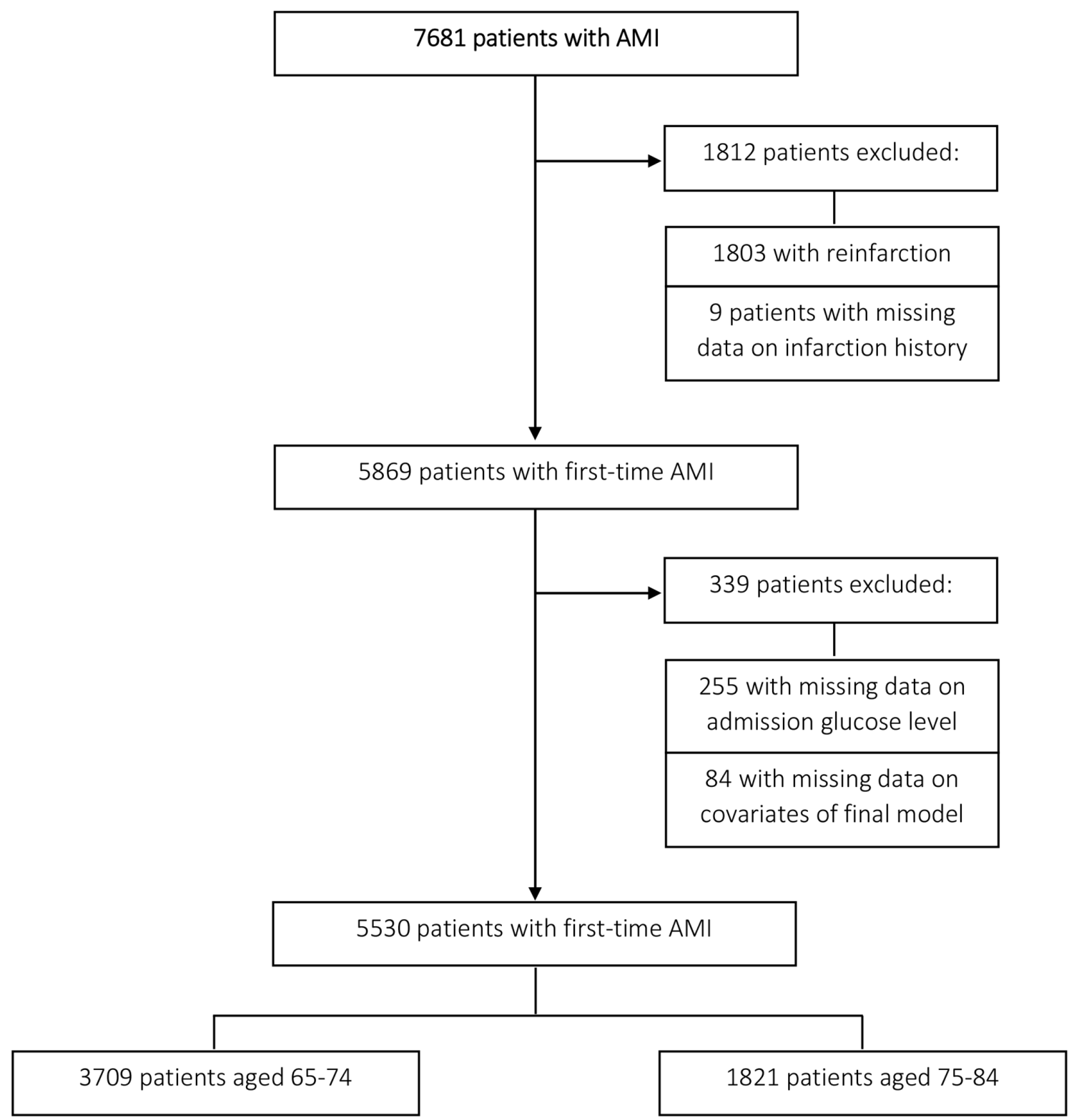

Figure 1 Flow chart diagram of study sample selection. Inclusion process for study sample with numbers and reasons for excluding patients from the original data set. AMI, acute myocardial infarction.

patients and a higher frequency of patients with a history of hypertension. In the younger age group, a higher prevalence of STEMI type as well as NSTEMI type than in the older age group was observed. Patients in the younger age group showed a higher prevalence of lipid disorders in comparison with the older age group.

Treatment during hospital stay according to the age groups is shown in table 2. More young-old patients less likely received ACE inhibitors, beta-blockers and nitrates. On the other hand, older patients were more often treated with calcium channel blockers and angiotensin II antagonists. There was no difference in treatment with lipid-lowering drugs, antiplatelets and insulin. At least one recanalisation therapy (PCI, CABG or thrombolysis) was more likely performed in the young-old compared with the older patients.
Major complications in patients with AMI occurring during hospital stay are listed in table 3. Frequency of in-hospital cardiac arrest was significantly higher in the older patients' group. Regarding other in-hospital complications including cardiogenic shock, pulmonary oedema, ventricular fibrillation, tachycardia and reinfarction there was no significant difference between the two age groups.

In the whole sample, as it is presented in table 4, admission blood glucose was significantly associated with 28-day case fatality: per 1 SD increase in admission blood glucose level the OR for 28-day mortality was 1.33 (95\% CI: 1.19 to 1.50$)$. In the young-old group there was also a significant relationship; per 1 SD increase of blood glucose the OR for 28-day case fatality was 1.40 (95\% CI: 1.21 to 1.62). Among the older patients, there was no significant 
Table 2 Treatment of patients with AMI during hospital stay by age groups

\begin{tabular}{|c|c|c|c|c|}
\hline & \multicolumn{2}{|l|}{ Age groups } & \multirow[b]{2}{*}{$P$ value } & \multirow{2}{*}{$\begin{array}{l}\text { Total sample } \\
(n=5530)\end{array}$} \\
\hline & $65-74(n=3709)$ & $75-84(n=1821)$ & & \\
\hline \multicolumn{5}{|l|}{ Drug treatment of patients with AMI } \\
\hline Ca-antagonists & $1343(36.2 \%)$ & $748(41.1 \%)$ & $<0.001$ & $2091(37.8 \%)$ \\
\hline ACE inhibitors & 3007 (81.1\%) & 1337 (73.4\%) & $<0.001$ & 4344 (78.5\%) \\
\hline Nitrates & $2948(79.5 \%)$ & $1237(67.9 \%)$ & $<0.001$ & $4185(75.7 \%)$ \\
\hline Angiotensin II antagonists & 404 (10.9\%) & $327(17.9 \%)$ & $<0.001$ & $731(13.2 \%)$ \\
\hline Other antihypertensives & $635(17.1 \%)$ & $395(21.7 \%)$ & $<0.001$ & $1030(18.6 \%)$ \\
\hline Statins & $3284(88.5 \%)$ & $1614(88.6 \%)$ & 0.956 & $4898(88.6 \%)$ \\
\hline Insulin & $1250(33.7 \%)$ & $610(33.5 \%)$ & 0.904 & $1860(33.6 \%)$ \\
\hline Bypass & $672(18.1 \%)$ & $236(13.0 \%)$ & $<0.001$ & $908(16.4 \%)$ \\
\hline Thrombolytic therapy & $160(4.3 \%)$ & $8(0.4 \%)$ & $<0.001$ & $168(3.0 \%)$ \\
\hline
\end{tabular}

AMI, acute myocardial infarction; $\mathrm{PCl}$, percutaneous coronary intervention.

association in the fully adjusted model (OR 1.21; $95 \%$ CI: 0.98 to 1.50$)$.

In addition, blood glucose levels at admission were independently associated with major in-hospital complications in the total sample and in both age groups (table 4). Among all patients, the OR for any major complication was 1.25 (95\% CI: 1.17 to 1.35 ) per 1 SD increase of blood glucose level; among the patients aged 65-74 years and 75-84 years, the ORs were 1.24 (95\% CI: 1.13 to 1.35 ) and 1.29 (95\% CI: 1.14 to 1.47 ) per 1 SD increase of blood glucose level, respectively.

Admission glucose level was significantly associated with higher 28-day mortality and hospital complications, irrespective of diabetes status in both the young-old and old groups (except the association with the 28-day mortality in the older group) (table 4). In patients with STEMI but not with NSTEMI, a significant association with 28-day case fatality could be observed for both young-old and older patients. Regarding in-hospital complications, in patients with STEMI, a significant relationship could be found for the older patients (OR 1.68; 95\% CI: 1.24 to 2.27). In patients with NSTEMI, a significant association with in-hospital complications could be shown for both the young-old (OR 1.17; 95\% CI 1.04 to 1.33 ) and old groups (OR 1.20; $95 \%$ CI 1.01 to 1.43 ).

\section{DISCUSSION}

In this real-world study including all consecutive hospitalised, unselected cases with incident AMI in patients 65-84 years of age, 28-day case fatality was associated with increasing blood glucose concentrations measured

Table 3 Complications in patients with AMI by age groups

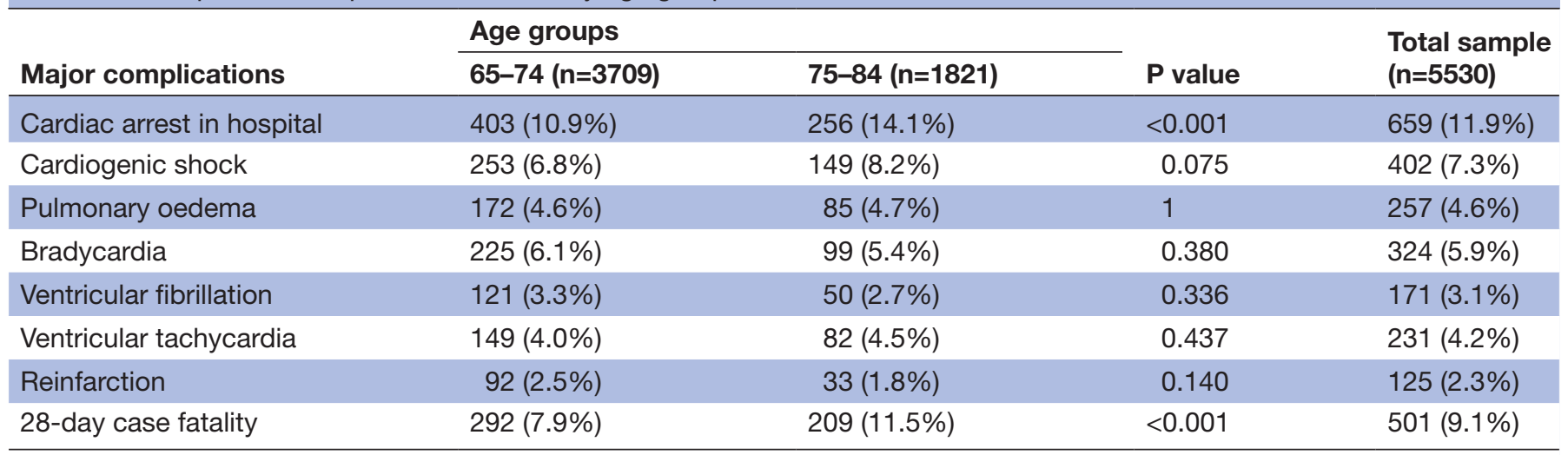

AMI, acute myocardial infarction. 


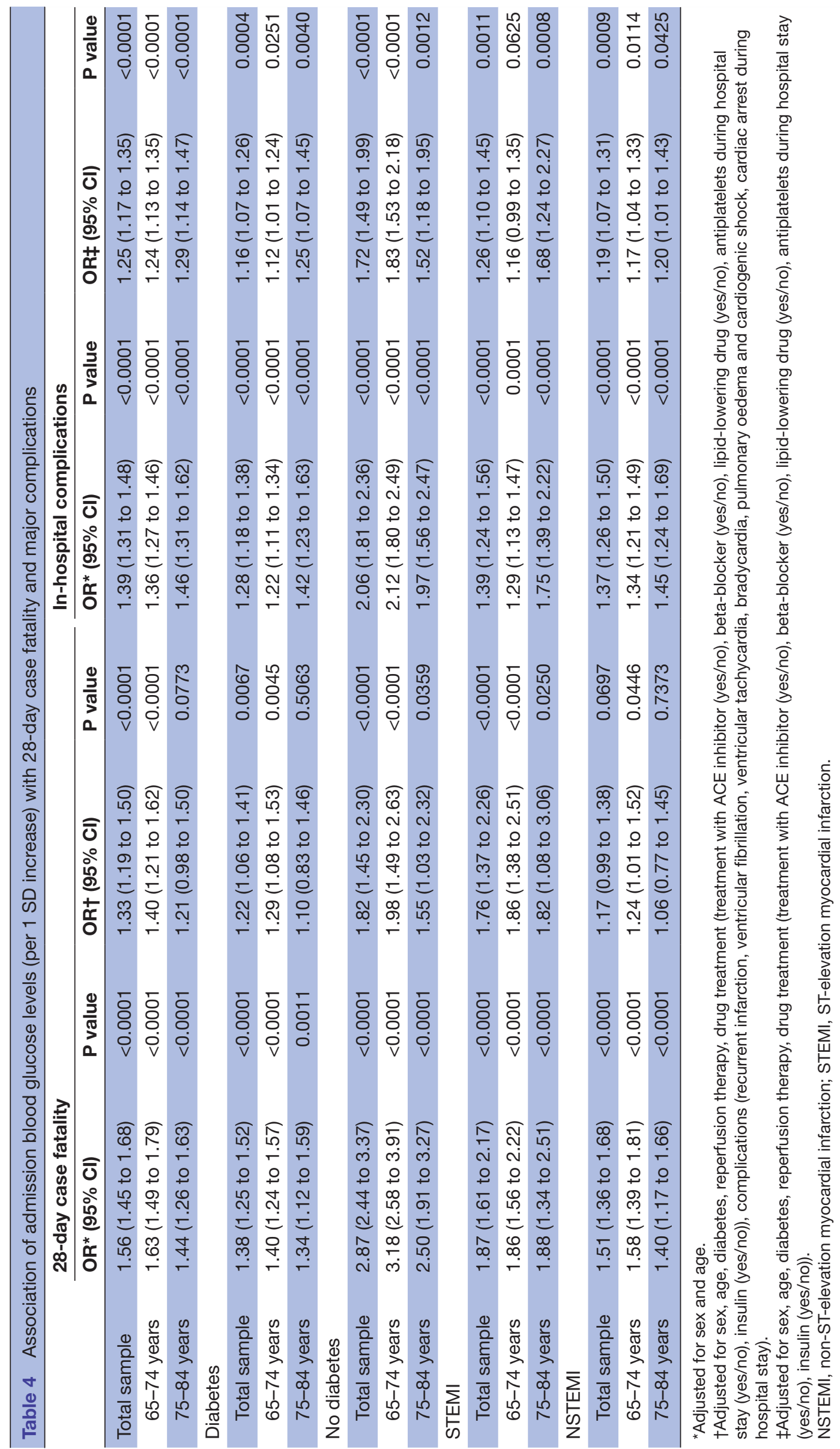


at hospital admission. The risk of death in the young-old patients (65-74 years) increased significantly with increasing blood glucose levels, but in the older patients' group (75-84 years) no independent association was found. In addition, admission glucose was significantly associated with a higher 28-day mortality in the total sample of patients with and without diabetes, and in patients with STEMI. The risk of major in-hospital complications after incident AMI was also related to higher admission blood glucose levels in both age groups, in the total sample of patients with and without diabetes, and patients with STEMI and NSTEMI.

Previous studies have demonstrated that elevated blood glucose on admission is common in patients with AMI and is independently associated with a higher risk of in-hospital mortality and in-hospital complications, such as cardiac arrest, cardiogenic shock and pulmonary oedema regardless of diabetes status. ${ }^{25-27}$ Although numerous studies have documented this association, ${ }^{2028-30}$ the impact of admission blood glucose on short-term mortality and in-hospital complications in older patients with AMI remains underappreciated so far.

In a large population-based study including patients with AMI aged 65 years and older, ${ }^{20}$ glucose levels were associated with 30-day case fatality in patients without known diabetes (referent: glucose $\leq 110 \mathrm{mg} / \mathrm{dL}$; range from glucose $>110-140 \mathrm{mg} / \mathrm{dL}$ : HR 1.17; $95 \%$ CI: 1.11 to 1.24 ; to glucose $>240 \mathrm{mg} / \mathrm{dL}$ : HR $1.87 ; 95 \%$ CI: 1.75 to 2.00). In a nationally representative study of patients (median age 67 years) hospitalised with AMI in China, Zhao et $a b^{0}$ reported that both moderate and severe hyperglycaemia (blood glucose $\geq 11.1 \mathrm{mmol} / \mathrm{L}$ ) on admission were associated with an elevated risk of in-hospital mortality among both patients without and with diabetes. Fujino $e t a l^{29}$ analysed the short-term outcome of acute hyperglycaemia on admission $(\geq 200 \mathrm{mg} / \mathrm{dL})$ and chronic hyperglycaemia defined by an HbAlc $\geq 6.5 \%$ in a small sample of patients with AMI and reported that acute hyperglycaemia but not chronic hyperglycaemia was an independent predictor of in-hospital mortality.

Several prior studies examined the association between hyperglycaemia on admission and complications of AMI. Dziewierz $e t a l^{25}$ analysed data of elderly patients with AMI of the Poland's Krakow Registry and found that hyperglycaemia on admission was related to an increased risk of pulmonary oedema and heart rhythm/conduction disturbances in both patients with and without diabetes. In another study, Kim $e t a l^{27}$ found a significant association between hyperglycaemia and life-threatening complications during hospitalisation such as cardiogenic shock, decreased haemoglobin level (haemoglobin $\geq 0.5 \mathrm{~g} / \mathrm{L}$ ), atrioventricular block, ventricular tachycardia and atrial fibrillation. Besides, they observed that a higher age of patients ( $\geq 75$ years), female sex, STEMI, low LV function, low revascularisation ratio, larger infarct size and inflammation were related to hyperglycaemia on admission.

The results of the present study confirm the findings regarding a strong association between admission blood glucose and short-term mortality as well as in-hospital complications in patients with AMI independent of diabetes status. Contrary to our study, prior studies did not evaluate how the relationship between admission glucose and outcomes varies between different age groups or other AMI subgroups in higher aged patients. The present study therefore expands the current understanding of the relevance of admission glucose regarding adverse outcomes in subgroups of older patients with AMI. Further studies on this issue are necessary to confirm or refute our findings.

The present data indicated that admission glucose had different impacts on adverse short-term outcomes in elderly patients with STEMI versus NSTEMI. Prior studies investigating the relevance of admission glucose on outcomes were mostly conducted in patients with STEMI $^{31-33}$ or included both patients with STEMI and NSTEMI $^{2034} 35$; only a few studies were conducted in NSTEMI samples. ${ }^{36}$ In addition, studies on this issue conducted in elderly patients with AMI are scarce. ${ }^{37}$ For example, a meta-analysis including six cohort studies reported that elevated admission glucose ( $\geq 6.1-11.1$ $\mathrm{mmol} / \mathrm{L})$ was significantly associated with short-term mortality in patients with STEMI without diabetes (RR 4.38; 95\% CI 3.23 to 5.94) ${ }^{38}$ In another study conducted in patients with NSTEMI undergoing PCI, admission blood glucose was a predictor of 30-day major adverse cardiovascular events (MACE), irrespective of diabetes status. ${ }^{36}$ Our results suggested that admission glucose might be a predictor of short-term mortality and in-hospital complications in patients with STEMI aged 65-84 years old, while in patients with NSTEMI it was associated with in-hospital complications only.

The increased mortality related to high admission glucose levels in patients with AMI has been linked to different pathophysiological mechanisms. There is evidence for the toxic effects of hyperglycaemia on cell function, because acute high blood glucose might induce oxidative stress, most likely via generation of free radicals. ${ }^{2}$ Moreover, hyperglycaemia inhibits metabolic processes in the myocardium and induces apoptosis in cardiomyocytes. Chang $e t a l^{89}$ showed an association between high glucose level and sFas serum levels, which is a valuable biomarker of the physiological response to ischaemia.

Stress hyperglycaemia in patients with MI could also be associated with adverse outcomes due to its ability to increase systemic inflammation and activation of stress responsive kinases. Recently, Marfella et $a l^{40}$ demonstrated an association between inflammatory markers and functional cardiac outcome in patients with an incident MI. In that study, hyperglycaemia was associated with amplified inflammatory immune reactions and worse functional cardiac outcome.

Moreover, hyperglycaemia is strongly associated with impaired coronary flow before reperfusion and has been related to enhanced thrombin formation, platelet activation and fibrin clot resistance to lysis. Hyperglycaemia has been linked to increased sensitivity to ischaemia 
reperfusion injury. ${ }^{51}$ These pathological processes may vary with age, that could explain, at least in part, our results.

It is well known that age is a risk factor for cardiovascular disease and an independent risk factor for mortality and adverse outcomes after AMI. For example, Shechter et $a t^{42}$ demonstrated that patients with AMI over 80 years had more MACE (including reinfarction, post-infarction angina, ischaemic stroke, high-degree atrioventricular block, acute renal failure and major bleeding) in-hospital and a fourfold to fivefold higher mortality rate than younger patients. Furthermore, age is related to frequent complications and side effects of treatment interventions and pharmacotherapy. ${ }^{43}$ Additionally, the haemodynamic impact of a given infarct size may be more pronounced in the elderly as a result of reduced cardiac reserve. ${ }^{44}$ There is also a greater likelihood of comorbid illnesses with advancing age, which contribute to poorer outcomes. ${ }^{45}$ The non-significant association between admission glucose levels and 28-day mortality in the age group 75-84 years in our study may be attributed to the fact that these patients suffered more often from comorbidities and were more severely ill (eg, higher complication rate, a higher proportion of patients with an LV ejection fraction $<30 \%$ ) compared with the younger age group; it might be thinkable that admission glucose values might not have a major influence on the case fatality in this group.

Several important limitations of the present study should be acknowledged. First, our study was observational and non-randomised by nature and therefore, causality could not be evaluated. Second, the analysis was limited to admission blood glucose values. In patients without diabetes, admission blood glucose alone without HbAlc values to test for undiagnosed diabetes or prediabetes and without post-discharge tests to assess the glycaemic state after the drop of stress during hospital admission, the meaning and interpretation of admission hyperglycaemia in clinical practice is difficult. ${ }^{46-49} \mathrm{We}$ cannot exclude the possibility that the outcome in the group without diabetes was driven by pre-diabetes or undiagnosed diabetes. Furthermore, there is a lack of information on the effect of in-hospital treatment regarding hyperglycaemia and hypoglycaemia, and how glucose levels during hospital stay affected adverse outcomes. Additionally, in our study, we did not assess major comorbidities, which can increase the risk of death (eg, lung disease, chronic renal failure or peripheral vascular disease) and for this reason our results should be interpreted with caution. Although our multivariable analysis was adjusted for several risk factors, residual confounding cannot be entirely excluded. Finally, our study was limited to German patients aged 65-84 years old with incident AMI, therefore it remains uncertain if our results apply to other populations and age subgroups of patients.

The present study is characterised by several strengths. Data were collected within the framework of a populationbased MI registry, and the consecutively admitted patients included from the general population presenting with first AMIs were registered according to a standardised protocol. Furthermore, important risk factors, in-hospital treatment, complications and types of infarction were included in our analysis.

\section{CONCLUSIONS}

Admission blood glucose was significantly associated with 28-day case fatality in patients with AMI aged 65-74 years but not 75-84 years; furthermore, in both age groups there was an increased risk of major complications. After stratification for diabetes and type of infarction, admission blood glucose was significantly related to case fatality irrespective of the diabetes status and type of infarction only in the patients aged $65-74$ years. Thus, it is likely that admission glucose plays only a minor role in terms of case fatality in higher aged patients with AMI. The older the patients are the more comorbidities they may have and the sicker these patients may be when admitted to hospital. The probability that these patients die from these conditions seems to be higher than that they die as a result of increased admission glucose.

\section{Author affiliations}

${ }^{1}$ Institute for Medical Information Processing, Biometry and Epidemiology (IBE), Ludwig-Maximilians-University of Munich, Munchen, Germany

${ }^{2}$ Chair of Epidemiology, UNIKA-T Augsburg, Ludwig-Maximilians-Universität München, Augsburg, Germany

${ }^{3}$ Institute of Epidemiology, Helmholtz Zentrum Munchen, German Research Center for Health and Environment, Neuherberg, Germany

${ }^{4}$ KORA Study Centre, University Hospital Augsburg, Augsburg, Germany ${ }^{5}$ Independent Research Group Clinical Epidemiology, Helmholtz Zentrum Munchen, German Research Center for Health and Environment, Neuherberg, Germany

${ }^{6}$ Department of Internal Medicine/Cardiology, Hospital of Nördlingen, Nördlingen, Germany

${ }^{7}$ Department of Cardiology, Respiratory Medicine and Intensive Care, University Hospital Augsburg, Augsburg, Germany

Acknowledgements The KORA Study was initiated and financed by the Helmholtz Zentrum München-German Research Center for Environmental Health, which is funded by the German Federal Ministry of Education and Research (BMBF) and by the State of Bavaria.

Contributors TM, KV and CM conceived the study. DF, TM and KV performed the statistical analysis and interpreted the results with feedback from $\mathrm{CM}, \mathrm{JL}, \mathrm{UA}, \mathrm{BK}$, $\mathrm{MH}, \mathrm{AP}$ and $\mathrm{CT}$. TM and KV drafted and revised the manuscript based on comments, which were provided by all authors. $\mathrm{CM}, \mathrm{MH}, \mathrm{BK}, \mathrm{AP}$ and $\mathrm{CT}$ contributed to data acquisition. All authors revised the manuscript critically for important intellectual content and approved the final version.

Funding This research was a master's thesis of TM and was not supported by a grant.

Competing interests None declared.

Patient and public involvement Patients and/or the public were not involved in the design, or conduct, or reporting, or dissemination plans of this research.

Patient consent for publication Obtained.

Ethics approval The study has been approved by the ethics committee of the Bavarian Medical Association (Ethik-Kommission Nr. 08064) and the study was performed in accordance with the Declaration of Helsinki. All study participants gave written informed consent.

Provenance and peer review Not commissioned; externally peer reviewed.

Data availability statement Data are not publicly available. Project agreements to use and access KORA data can be requested from national and international researchers via the KORA-PASST tool under https://epi.helmholtz-muenchen.de/ 
Open access This is an open access article distributed in accordance with the Creative Commons Attribution Non Commercial (CC BY-NC 4.0) license, which permits others to distribute, remix, adapt, build upon this work non-commercially, and license their derivative works on different terms, provided the original work is properly cited, appropriate credit is given, any changes made indicated, and the use is non-commercial. See: http://creativecommons.org/licenses/by-nc/4.0/.

\section{ORCID iD}

Temur Mamadjanov http://orcid.org/0000-0002-7241-5702

\section{REFERENCES}

1 Goyal A, Mehta SR, Gerstein HC, et al. Glucose levels compared with diabetes history in the risk assessment of patients with acute myocardial infarction. Am Heart J 2009;157:763-70.

2 Kosiborod M, Inzucchi SE, Krumholz HM, et al. Glucometrics in patients hospitalized with acute myocardial infarction: defining the optimal outcomes-based measure of risk. Circulation 2008;117:1018-27.

3 Capes SE, Hunt D, Malmberg K, et al. Stress hyperglycaemia and increased risk of death after myocardial infarction in patients with and without diabetes: a systematic overview. Lancet 2000;355:773-8.

4 Goyal A, Mahaffey KW, Garg J, et al. Prognostic significance of the change in glucose level in the first $24 \mathrm{H}$ after acute myocardial infarction: results from the cardinal study. Eur Heart $J$ 2006;27:1289-97.

5 Timmer JR, Ottervanger JP, de Boer M-J, et al. Hyperglycemia is an important predictor of impaired coronary flow before reperfusion therapy in ST-segment elevation myocardial infarction. J Am Coll Cardiol 2005;45:999-1002.

6 Timmer JR, Hoekstra M, Nijsten MWN, et al. Prognostic value of admission glycosylated hemoglobin and glucose in nondiabetic patients with ST-Segment-Elevation myocardial infarction treated with percutaneous coronary intervention. Circulation 2011;124:704-11.

7 Timmer JR, van der Horst ICC, Ottervanger JP, et al. Prognostic value of admission glucose in non-diabetic patients with myocardial infarction. Am Heart J 2004;148:399-404.

8 Norhammar AM, Rydén L, Malmberg K. Admission plasma glucose. independent risk factor for long-term prognosis after myocardial infarction even in nondiabetic patients. Diabetes Care 1999;22:1827-31.

9 Iwakura K, Ito $\mathrm{H}$, Ikushima $\mathrm{M}$, et al. Association between hyperglycemia and the no-reflow phenomenon in patients with acute myocardial infarction. J Am Coll Cardiol 2003;41:1-7.

10 Cheng $\mathrm{H}-\mathrm{H}$, Yen $\mathrm{P}-\mathrm{C}$. Killip classification and glucose level in patients with acute myocardial infarction. Am J Emerg Med 2010;28:853-6.

$11 \mathrm{Hsu} \mathrm{C}-\mathrm{W}$, Chen $\mathrm{HH}$, Sheu WH-H, et al. Initial serum glucose level as a prognostic factor in the first acute myocardial infarction. Ann Emerg Med 2007;49:618-26.

12 Correia LCL, Rocha MS, Bittencourt AP, et al. Does acute hyperglycemia add prognostic value to the grace score in individuals with non-ST elevation acute coronary syndromes? Clin Chim Acta 2009;410:74-8

13 Timóteo AT, Papoila AL, Rio P, et al. Prognostic impact of admission blood glucose for all-cause mortality in patients with acute coronary syndromes: added value on top of grace risk score. Eur Heart $J$ Acute Cardiovasc Care 2014;3:257-63.

14 Bellodi G, Manicardi V, Malavasi V, et al. Hyperglycemia and prognosis of acute myocardial infarction in patients without diabetes mellitus. Am J Cardiol 1989;64:885-8.

15 O'Sullivan JJ, Conroy RM, Robinson K, et al. In-Hospital prognosis of patients with fasting hyperglycemia after first myocardial infarction. Diabetes Care 1991;14:758-60.

16 Oswald GA, Smith CC, Betteridge DJ. Determinants and importance of stress hyperglycaemia in non-diabetic patients with myocardial infarction.. In: Br Med J, ed, 1986: 917-22.

17 Hadjadj S, Coisne D, Mauco G, et al. Prognostic value of admission plasma glucose and $\mathrm{HbA}$ in acute myocardial infarction. Diabet Med 2004;21:305-10.

18 Sala J, Masiá R, González de Molina F-J, et al. Short-Term mortality of myocardial infarction patients with diabetes or hyperglycaemia during admission. J Epidemiol Community Health 2002;56:707-12.

19 Foo K, Cooper J, Deaner A, et al. A single serum glucose measurement predicts adverse outcomes across the whole range of acute coronary syndromes. Heart 2003;89:512-6.

20 Kosiborod M, Rathore SS, Inzucchi SE, et al. Admission glucose and mortality in elderly patients hospitalized with acute myocardial infarction: implications for patients with and without recognized diabetes. Circulation 2005;111:3078-86.

21 Kuch B, Heier M, von Scheidt W, et al. 20-Year trends in clinical characteristics, therapy and short-term prognosis in acute myocardial infarction according to presenting electrocardiogram: the MONICA/KORA AMI registry (1985-2004). J Intern Med 2008;264:254-64.

22 Löwel $\mathrm{H}$, Meisinger $\mathrm{C}$, Heier $\mathrm{M}$, et al. The population-based acute myocardial infarction (AMI) registry of the MONICA/KORA study region of Augsburg. Gesundheitswesen 2005;67 Suppl 1:31-7.

23 Meisinger C, Hörmann A, Heier M, et al. Admission blood glucose and adverse outcomes in non-diabetic patients with myocardial infarction in the reperfusion era. Int J Cardiol 2006;113:229-35.

24 Alpert JS, Thygesen K, Antman E, et al. Myocardial infarction redefined--a consensus document of The Joint European Society of Cardiology/American College of Cardiology Committee for the redefinition of myocardial infarction. J Am Coll Cardiol 2000;36:959-69.

25 Dziewierz A, Giszterowicz D, Siudak Z, et al. Admission glucose leve and in-hospital outcomes in diabetic and non-diabetic patients with acute myocardial infarction. Clin Res Cardiol 2010;99:715-21.

26 Ishihara M. Acute hyperglycemia in patients with acute myocardial infarction. Circ J 2012;76:563-71.

$27 \mathrm{Kim}$ EJ, Jeong MH, Kim JH, et al. Clinical impact of admission hyperglycemia on in-hospital mortality in acute myocardial infarction patients. Int J Cardiol 2017;236:9-15.

28 Ding XS, Wu SS, Chen $\mathrm{H}$, et al. High admission glucose levels predict worse short-term clinical outcome in non-diabetic patients with acute myocardial infraction: a retrospective observational study. BMC Cardiovasc Disord 2019;19:163

29 Fujino M, Ishihara M, Honda S, et al. Impact of acute and chronic hyperglycemia on in-hospital outcomes of patients with acute myocardial infarction. Am J Cardiol 2014;114:1789-93.

30 Zhao S, Murugiah K, Li N, et al. Admission glucose and inhospital mortality after acute myocardial infarction in patients with or without diabetes: a cross-sectional study. Chin Med J 2017;130:767-75.

31 Li D-bao, Hua Q, Guo J, et al. Admission glucose level and inhospital outcomes in diabetic and non-diabetic patients with STelevation acute myocardial infarction. Intern Med 2011;50:2471-5.

32 Chen P-C, Chua S-K, Hung H-F, et al. Admission hyperglycemia predicts poorer short- and long-term outcomes after primary percutaneous coronary intervention for ST-elevation myocardial infarction. J Diabetes Investig 2014;5:80-6.

33 Pinto DS, Kirtane AJ, Pride YB, et al. Association of blood glucose with angiographic and clinical outcomes among patients with STsegment elevation myocardial infarction (from the CLARITY-TIMI-28 study). Am J Cardiol 2008;101:303-7.

34 Norhammar A, Tenerz A, Nilsson G, et al. Glucose metabolism in patients with acute myocardial infarction and no previous diagnosis of diabetes mellitus: a prospective study. Lancet 2002;359:2140-4.

35 Stranders I, Diamant M, van Gelder RE, et al. Admission blood glucose level as risk indicator of death after myocardial infarction in patients with and without diabetes mellitus. Arch Intern Med 2004;164:982-8.

36 Hao Y, Lu Q, Li T, et al. Admission hyperglycemia and adverse outcomes in diabetic and non-diabetic patients with non-STelevation myocardial infarction undergoing percutaneous coronary intervention. BMC Cardiovasc Disord 2017;17:6.

37 Otten AM, Ottervanger JP, Timmer JR, et al. Age-Dependent differences in diabetes and acute hyperglycemia between men and women with ST-elevation myocardial infarction: a cohort study. Diabetol Metab Syndr 2013;5:34.

38 Zhao C-jin, Hao Z-xuan, Liu R, et al. Admission glucose and risk of early death in non-diabetic patients with ST-segment elevation myocardial infarction: a meta-analysis. Med Sci Monit 2015;21:1387-94.

39 Chang J, Zhang G, Zhang L, et al. High admission glucose levels increase Fas apoptosis and mortality in patients with acute ST-elevation myocardial infarction: a prospective cohort study. Cardiovasc Diabetol 2013;12:171.

40 Marfella R, Siniscalchi M, Esposito K, et al. Effects of stress hyperglycemia on acute myocardial infarction: role of inflammatory immune process in functional cardiac outcome. Diabetes Care 2003;26:3129-35.

41 Undas A, Wiek I, Stêpien E, et al. Hyperglycemia is associated with enhanced thrombin formation, platelet activation, and fibrin clot resistance to lysis in patients with acute coronary syndrome. Diabetes Care 2008;31:1590-5.

42 Shechter M, Rubinstein R, Goldenberg I, et al. Comparison of Outcomes of Acute Coronary Syndrome in Patients $\geq 80$ Years 
Versus Those $<80$ Years in Israel from 2000 to 2013. Am J Cardiol 2017;120:1230-7.

43 Stone $\mathrm{PH}$, Thompson B, Anderson HV, et al. Influence of race, sex, and age on management of unstable angina and non-Q-wave myocardial infarction: the TIMI III registry. JAMA 1996;275:1104-12.

44 De Carlo M, Morici N, Savonitto S, et al. Sex-Related outcomes in elderly patients presenting with non-ST-segment elevation acute coronary syndrome: insights from the Italian elderly ACS study. JACC Cardiovasc Interv 2015;8:791-6.

45 Gudnadottir GS, James SK, Andersen K, et al. Outcomes after STEMI in old multimorbid patients with complex health needs and the effect of invasive management. Am Heart J 2019;211:11-21.

46 Clement S, Braithwaite SS, Magee MF, et al. Management of diabetes and hyperglycemia in hospitals. Diabetes Care 2004;27:553-91.
47 Handelsman Y, Bloomgarden ZT, Grunberger G, et al. American association of clinical endocrinologists and american college of endocrinology - clinical practice guidelines for developing a diabetes mellitus comprehensive care plan - 2015. Endocr Pract 2015;21 Suppl 1:1-87.

48 Chattopadhyay S, George A, John J, et al. Two-Hour post-challenge glucose is a better predictor of adverse outcome after myocardial infarction than fasting or admission glucose in patients without diabetes. Acta Diabetol 2018;55:449-58.

49 Chattopadhyay S, George A, John J, et al. Newly diagnosed abnormal glucose tolerance determines post-Ml prognosis in patients with Hospital related hyperglycaemia but without known diabetes. J Diabetes Complications 2020;34:107518. 